\title{
Une esthétique du compromis : discours métalinguistique et subversion dans L'Euguélionne de Louky Bersianik
}

\author{
Sophie Coupal
}

Louky Bersianik's L'Euguélionne (1976) invites women to dismantle a phallocentric symbolic order through the transgression of linguistic codes. But while Bersianik pursues a questioning of language at the level of discourse, this questioning does not reach to the level of novelistic form. Bersianik's project is thus characterized by an aesthetic of compromise that negotiates between generic exigencies and a concern for accessibility - hesitating to subject language to a more radical subversion.

Il est pratiquement impossible d'ignorer l'extraordinaire effervescence qui marque la production féminine québécoise dans le domaine de la création littéraire à partir de 1975. La montée du féminisme radical depuis la fin des années soixante et la prolifération des mouvements de femmes préparent le terrain et annoncent de chaudes luttes en faveur de changements sociaux. Les femmes refusent désormais de se taire et l'écriture représente bien souvent le moyen privilégié de faire entendre leurs voix.

Cependant, à l'euphorie générée par la prise de parole se mêle bien souvent, chez les femmes auteures, la douloureuse conscience qu'il ne suffit pas seulement, pour écrire, d'avoir une chambre à soi. La langue elle-même devient rapidement une cible de choix pour les féministes de toutes origines. Certaines écrivaines québécoises, se sentant à l'étroit dans la langue ou carrément trahies par un système de communication qui les rejette, entreprennent alors un travail de transgression qui les mènera ultimement à l'inscription de la subjectivité féminine dans la langue. Louky Bersianik est probablement l'auteure à s'être la plus illustrée, de pair avec Nicole Brossard, Madeleine Gagnon et France Théoret, dans ce courant de redéfinition des pratiques langagières des femmes dans l'écriture. L'Euguélionne, publié en 1976, est un parfait exemple du rapport tendu que Bersianik entretient avec sa langue. Considéré par plusieurs comme la « Bible des femmes » (Bersianik, « La mémoire courte » 50), ce « roman tryptique » se caractérise par un mélange des genres où le 
roman, le manifeste et le traité de linguistique cohabitent et s'interpénètrent. Les nombreuses récriminations en matière de langue que le volume contient ne se répercutent que faiblement au niveau de la forme, révélant par là une esthétique du compromis qui sous-tend le texte, responsable à la fois de ses forces et de ses faiblesses. Représentative du rapport particulier de l'écrivaine aux contraintes du code linguistique, cette esthétique se définit entre la nécessité, pressentie par l'auteure, d'une subversion efficace de la langue et le choix d'une écriture accessible au grand public. Les stratégies linguistiques de détournement mises en place dans L'Euguélionne sont traversées avant tout par un souci de "légalité " (Bersianik, "Langue étrangère » 170) qui souligne à grand trait les tensions s'établissant au sein de l'écriture lorsqu'une femme décide de se mettre à parler en refusant de souscrire à ce que les féministes ont identifié comme étant une norme de domination masculine de la langue. Cette ambivalence de Bersianik à subvertir le code linguistique de façon radicale, comme par exemple, à la même époque, Nicole Brossard, révèle l'extraordinaire difficulté d'une écriture qui se forme à même ce qu'elle tente de déconstruire. Afin de mettre cette oppression en relief, je propose d'abord un tour d'horizon du discours métalinguistique chez Bersianik, pour ensuite voir comment il se répercute au niveau de la forme dans L'Euguélionne. En comparant les aspects théorique et pratique, je devrais être à même de faire pressentir toute la richesse de la tension qui règne au sein de L'Euguélionne entre le désir et le faire.

Le désir de subversion est d'abord généré chez Bersianik par le sentiment douloureux d'une injustice. La langue est perçue par l'auteure comme un «gag » qui se joue tous les jours aux dépens des femmes:

La langue pour moi, le langage c'est un immense gag. Alors je pourrais dire qu'en tant que femme je suis née du mauvais bord de la langue parce que je suis née du bord de la langue du mépris. Et c'est comme si on est dans un éclat de rire général et on ne s'aperçoit pas qu'on en fait les frais, nous autres les femmes, de cet éclat de rire général. (Bersianik, « Transcription d'une émission de radio » 6)

De gag à langue, Bersianik fait un de ces mots-valises qu'elle affectionne tout particulièrement : gangue. 
Le passé du langage est un gag.

Au présent, la langue dort dans une gangue

comme dans ses langes.

L'avenir est une formidable gageure.

Un gag / une gageure

Un langage / une gangue

$[\ldots]^{1}$

Comme le mot gangue existe déjà, ses définitions usuelles viennent s'ajouter au lien que Bersianik fait entre la langue et une plaisanterie. L'auteure met à profit toutes les définitions du mot : « 1 . Substance stérile engagée dans le minerai ou qui entoure une pierre précieuse dans un gisement. 2. Fig. Ce qui enveloppe, dissimule quelque chose »(Petit Larousse). La gangue enveloppe le " caillou de barbarie " qu'il faut " polir avant de commencer à dire quoi que ce soit » (MT 64). $\mathrm{D}^{\prime}$ apparence innocente, ce petit poème est pourtant pensé au mot près. Le jeu entre le féminin et le masculin accentue la dissymétrie qui affecte les deux sexes : au féminin sont associés les mots gageure et gangue, alors qu'au masculin, l'auteure lie gag et langage. La parole, comme le rire, sont l'apanage de l'homme. Pour la femme, il ne demeure qu'une langue dénaturée, "substance stérile », et la « formidable gageure » du futur, qui indique qu'on entend bien ne pas en rester là.

Cette notion de « gangue » des femmes, succédané du « langage » des hommes, empêche les femmes d'accéder au coeur de la langue. Celles-ci sont alors confinées au silence par l'action du « Verbe-Mâle » :

Quand le Verbe se fait Chair ressuscitée on l'entend dire : Noli me tangere

Je suis intouchable, moi, le Verbe-Mâle parce que je suis la loi

Et la loi c'est la loi / parce que la loi c'est moi, dit le Verbe-Mâle

Le reste est Histoire sans Parole. (« Noli mi tangere » 150)

L'association entre l'homme et la parole est tout à fait claire : la femme destinée au mutisme, $l^{\prime}$ « Histoire sans Parole », est placée en retrait par rapport au tout autosuffisant que forment $l^{\prime}$ Homme, son Dieu, sa Parole et sa Loi. Les femmes demeurent prisonnières de la « gangue ». 
Que nous le voulions ou non, nous sommes prises au piège de cette gangue qui enveloppe la langue dès qu'elle se parle au féminin : nous sommes parlées et gangrenées par elle. Là où elle est un joyau pour les académiciens et les intellectuels mâles, cette langue n'est pour nous femmes, et nous écrivaines, qu'une pierre hostile et sans éclat, qu'un caillou de barbarie, que nous devons polir avant de commencer à dire quoi que ce soit. (MT 64)

La langue qu'elles ont apprise constitue pour elles une source d'aliénation puisque, selon Lacan, que Bersianik qualifie de «fin-finaud» (Smith 68) : «Il n'y a de femme qu'exclue par la nature des choses qui est la nature des mots, et il faut bien dire que s'il y a quelque chose dont elles-mêmes se plaignent assez pour l'instant, c'est bien de ça - simplement, elles ne savent pas ce qu'elles disent, c'est toute la différence entre elles et moi » (Lacan 94). Sont exprimés ainsi en termes condescendants les effets de distorsion causés par cette " gangue » qui, en coupant la femme de ses propres perceptions (elle n'a pas de mots pour les exprimer), la force à faire sienne la vision masculine du monde à travers l'utilisation de la " langue de l'occupant » :

Ils nous ont ouvert la bouche de nouveau et nous ont greffé une langue étrangère. Et la greffe a pris.

Pas de rejet. Nous n'avons pas rejeté la langue de l'occupant.

Ils nous ont enfoncé des mots et des règles de grammaire dans la bouche. Et nous avons commencé à parler. (MT 46)

Pour Bersianik, c'est cette inadéquation entre leurs sentiments, leurs expériences et les moyens pour les exprimer qui rend les femmes complètement aliénées au sein de la langue. Le fait, pour les femmes, d'utiliser " la langue de l'occupant ", équivaut à reconnaître et à consacrer leur état de domination par les hommes. Utiliser la langue sans la "polir » auparavant revient à l'acceptation de tout ce qui s'y trouve en termes de charge négative contre les femmes. Pierre Bourdieu analyse ce phénomène dans son ouvrage intitulé La domination masculine:

Lorsque les dominés appliquent à ce qui les domine des schèmes qui sont le produit de la domination, ou, en d'autres termes, lorsque leurs pensées et leurs perceptions sont structurées conformément aux 
structures mêmes de la relation de domination qui leur est imposée, leurs actes de connaissance sont, inévitablement, des actes de reconnaissance, de soumission. (Bourdieu 19)

$C^{\prime}$ est ce qui amène Bersianik à revendiquer «l'incompétence linguistique » (MT 56) des femmes (d'après le concept de compétence linguistique de Chomsky), puisque de toute façon, leurs propres expériences ne sont pas inclues dans les définitions des mots qu'on retrouve dans les dictionnaires:

Cette compétence, donc, c'est l'intuition particulière que tout individu possède de sa propre langue et des moyens de l'utiliser. La personne qui a acquis la connaissance d'une langue, toujours selon Chomsky, a assimilé un système de règles reliant d'une certaine façon le son et le sens. Et c'est bien là que le bât blesse. Pour une femme, quel rapport y a-t-il entre les sons " homme », " travail », " esquimaux ", " pionnier ", et leur sens respectif? Même si ces mots sont censés la représenter elle aussi, elle n'y voit là que du mâle ... tout comme le mâle n'y voit que luimême, car il sait qu'il est la règle normative de l'humanité. (MT 57)

Bersianik amène la lectrice (ou le lecteur) à se poser la question ainsi formulée par Louise Forsyth : «Est-ce que le référent du signe coïncide nécessairement avec le monde extérieur, comme on le croit d'ordinaire? » (Forsyth 179). Pour toute réponse, Bersianik désigne l'écart entre le bagage (masculin) récolté par les mots au cours de l'histoire et la réalité telle que perçue par les femmes : «Les conventions linguistiques, les femmes ne peuvent plus les admettre, car elles sont une manifestation inconsciente de la croyance en la supériorité masculine et des conséquences de cette croyance : domination, mépris, violence » (MT 58). Pour Bersianik, « la langue de l'occupant », lorsque greffée dans la bouche de l'occupée, produit des effets de distorsion qui empêchent la coïncidence des sentiments et des paroles. $^{2}$

Devant cette aliénation de la femme au sein de la langue, comment s'en sortir ? Pour Bersianik, «transgresser, c'est progresser » (L'Euguélionne 211) : « En tant qu'écrivaines et en tant que femmes, nous devons développer des stratégies linguistiques "peccamineuses" en pensées, en paroles et en actes autant qu'en écriture, et répandre joyeusement le mauvais exemple qui fera grincer les puristes » (MT 64). L'adjectif « peccamineuses», terme de l'ancienne médecine (XVII ${ }^{\mathrm{e}}$ siècle) repris en 1884 par Huysmans dans 
son sens religieux de " nature du péché ${ }^{3}$ », est employé ici par Bersianik dans une allusion au discours religieux qui, faut-il le rappeler, est une des cibles principales de la critique dans L'Euguélionne. Les stratégies linguistiques subversives se rapprochent donc, dans l'esprit de l'auteure, d'un " péché » commis contre l'autorité patriarcale. Le seul fait d'employer cette expression désuète est révélateur de la tension entre désir et faire chez Bersianik. Celle-ci a conscience de s'aventurer en terrain interdit. De peccamineuses, aujourd'hui disparu des dictionnaires, on pourrait aisément faire un mot-valise, formé à partir du nom commun peccadille ("Faute sans gravité " (Petit Robert)) et du verbe miner ("Creuser, attaquer la base ou l'intérieur de (une chose) " ou encore "Attaquer, affaiblir, ruiner par une action progressive et sournoise" (Petit Robert)). Le terme prendrait alors la valeur d'un concept dans la pratique d'écriture de Bersianik, où les stratégies linguistiques de détournement sont en effet à voir comme des « péchés mineurs », visant, par une action progressive, à « miner » la Loi patriarcale. On sent d'ailleurs la pression de cette dernière dans la déclaration suivante de l'auteure : « Bien sûr, j'ai développé des stratégies linguistiques légales de détournement » ("Langue étrangère » 170; je souligne). La définition de la pratique d'écriture de Bersianik passe par son rapport à la Loi. Ce qui est frappant, dans les dernières citations, c'est de voir à quel point les moyens effectifs de subversion du langage que Bersianik se propose d'employer semblent peu puissants par rapport à l'ardeur des dénonciations et des réclamations en matière de langue.

Dans la pratique d'écriture de Bersianik, le discours métalinguistique s'articule étroitement avec la critique sociale. La langue est perçue principalement comme une instance de consécration du sexisme qui règne dans nos sociétés, l'inscrivant en son sein et participant ainsi à sa propagation. Les récriminations en matière de langue sont nombreuses dans L'Euguélionne et se regroupent principalement au début de la troisième partie. Elles concernent essentiellement le pouvoir de nommer qui a été nié aux femmes depuis la Création. À Roland Barthes qui rêve, dans Le degré zéro de l'écriture, d'un langage « dont la fraîcheur, par une sorte d'anticipation idéale, figurerait la perfection d'un nouveau monde adamique où le langage ne serait plus aliéné » (64-65), Bersianik réplique : "J'ai le regret de dire au regretté $\mathrm{M}$. Barthes que ce "monde adamique" où Adam seul nommait les choses et les gens, ce monde dont il rêve est justement celui dont les femmes ne veulent plus car c'est grâce à ce pouvoir de nommer qu'Adam les a dominées » (MT 57). 
Le pouvoir de nommer des hommes a fait couler beaucoup d'encre chez les féministes, notamment chez les Américaines, où une Dale Spender et une Mary Daly éprouvent le même sentiment de dépossession devant un pouvoir crucial de l'homme qui, ayant été enlevé à la femme depuis si longtemps, a effacé toute contribution de celle-ci au façonnement de l'histoire de l'humanité.

It is necessary to grasp the fundamental fact that women have had the power of naming stolen from them. We have not been free to use our own power to name ourselves, the world, or God. The old naming was not the product of a dialogue - a fact inadvertently admitted in the Genesis myth in which Adam names the animals and women. Women are now realising that this imposing of words was false because partial. That is, partial and inadequate words have been taken as adequate. ${ }^{4}$

Même si Bersianik n'était pas encore familière, à l'époque de la rédaction de L'Euguélionne, avec les travaux de Mary Daly, ${ }^{5}$ on remarque de fortes concordances entre leurs manières de penser. Le pouvoir de nommer est particulièrement important dans L'Euguélionne puisque, pour Bersianik, « [c]e qui n'est pas nommé n'existe pas » (MT 50). L'enjeu pour les femmes est de pouvoir imprimer leur marque sur une vision du monde qui a depuis toujours été dominée par un regard masculin. C'est là également l'opinion de Dale Spender : "In order to live in the world, we must name it. Names are essential for the construction of reality for without a name it is difficult to accept the existence of an object, an event, a feeling " (163). Le lien entre le langage et le monde est crucial. Cependant, pour ces auteures, comme la langue a été modelée par les hommes souvent au détriment des femmes, celles-ci entendent bien reconquérir leur place au sein $\mathrm{du}$ système symbolique. Le tout est d'arriver à se faire voir en déjouant les stratégies linguistiques masculines conçues pour gommer le féminin.

Le travail sur la langue dans L'Euguélionne est pensé de manière spécifique pour respecter les exigences de la communication. Les contraintes qui en découlent servent de moteur à l'écriture, à « la mise en mots, puis la mise en texte, selon certaines règles » («Entrevue » 72). La grande majorité des contraintes que s'impose l'auteure sont reliées à un objectif de cohérence et de constitution d'un tissu textuel très serré : " Pour moi, la première 
acception du mot texte, c'est son appellation latine : textus qui signifie tissu, trame, d'où enchaînement d'un récit, ou texte. Le mot latin texere veut dire tisser » («Entrevue » 68). La base de la technique d'écriture de Bersianik est constituée de ce qu'elle appelle « la translinéarité », qui vient croiser la « linéarité » (70) d'un texte, soit son sens littéral. « La translinéarité est faite de tous les éléments non contigus et significatifs d'un texte, éléments formant des réseaux d'association, soit par l'intermédiaire des idées, soit par liaison graphique ou sonore » (70). La «translinéarité »c'est donc l'axe paradigmatique qui traverse le texte à la verticale et auquel la trame narrative vient se mêler afin de créer le tissu textuel. On voit que tout dépend de l'idée de réseaux textuels, destinés à renforcer la cohérence interne du texte par un équilibre entre lisible et visible, linéarité et translinéarité, signifiant et signifié, syntagmatique et paradigmatique.

Cette préoccupation, jointe à un désir d'accessibilité et de communication des idées de l'auteure (dans le cadre de L'Euguélionne), nécessite un compromis entre un travail de la langue trop audacieux et un texte axé uniquement sur l'aspect discursif. Il faut faire échec à la tentation de l'illisibilité. C'est pourquoi Bersianik refuse de changer trop radicalement le cadre de références de ses lectrices et lecteurs. La parodie devient alors un moyen très utile pour opérer une transgression tout en conservant un lien avec le cadre conceptuel de la lectrice ou du lecteur moyen(ne). L'auteure ne vise pas un simple renversement du discours, mais bien une prise de conscience.

Transposé au niveau du travail sur la langue, ce compromis se traduit dans L'Euguélionne par la mise de côté relative d'une subversion grammaticale au profit de la transgression des règles de l'orthographe. En effet, si la question de la grammaire est assez bien traitée au niveau du discours (règle qui veut que le masculin l'emporte toujours sur le féminin et règles de formation des noms professionnels (L'Euguélionne 230-35)), elle reste plutôt lettre morte au plan de la forme. Bersianik n'a que très peu recours aux néologismes dont elle recommande elle-même l'utilisation. Dans un passage-clé du roman, l'Euguélionne enjoint les femmes à enfreindre les règles du français afin de s'assurer une place au sein de la langue : «Faites des fautes volontairement pour rétablir l'équilibre des sexes » (230). Elle a également ses propres idées sur la formation de féminins aux noms professionnels :

Je comprends vos réticences à former des féminins aux noms professionnels, car le féminin n'étant qu'une annexe au masculin, a 
toujours un sens moins sérieux quand il n'est pas carrément péjoratif. [...] Cependant, vous pouvez chercher dans l'étymologie du français à former vous-mêmes les substantifs propres à vous désigner dans vos professions. (L'Euguélionne 230)

Le " tableau des féminins en formation » que l’Euguélionne présente à la foule propose les mots suivants comme des équivalents féminins possibles du nom " auteur ", basés sur son étymologie : "Une auteure, Une croissante, Une augustine, Une augustelle, Une autoriste, Une auctorine, Une auctorèse, [...] Une automne, Une autelle, Une autesse » (231). Même si ces mots peuvent sembler " barbares », de l'aveu de l'Euguélionne, elle encourage les femmes à les utiliser le plus souvent possible afin que les meilleurs d'entre eux entrent dans l'usage. Cependant, à une quarantaine de pages de là, on remarque les constructions suivantes : « les recettes dont elles étaient les auteurs » (255) et « Soyez [femmes de la Terre] les auteurs à part entière de votre contrat social. » (292). Le fait que Bersianik laisse de côté ses propres néologismes au profit d'une langue standard en dit long sur le compromis qu'elle s'impose afin de préserver la clarté de son texte, de même que son autorité en matière de langue. Car pour arriver à subvertir efficacement la langue, encore faut-il prouver qu'on la maîtrise.

C'est en partie afin d'assurer sa compétence linguistique que Bersianik va systématiquement accentuer presque toutes ses innovations langagières à l'aide d'italiques, de guillemets, de majuscules, etc. C'est que la subversion de la langue comporte le même risque que l'ironie pour l'auteure : elle risque de ne pas être comprise. Il faut en effet que la subversion soit perçue comme telle et non comme une faute par la lectrice ou le lecteur. $\mathrm{Si}$ l'auteure se permet à l'occasion une féminisation « illégale », elle prend bien la peine de souligner, par l'italique, qu'elle est consciente de sa « faute » et qu'elle la fait par principe et en toute connaissance de cause : «Telle, [...] première députée de l'espèce pédaleuse à être élue » (L'Euguélionne 22). Cette tyrannie de la langue qui oblige l'écrivaine à respecter le code est à mon avis ce qui représente l'obstacle le plus lourd pour Bersianik. Dix ans plus tard, son déchirement est toujours évident :

Je suis encore avec elle [la langue] à couteaux tirés. Elle, avec ses coups de langue, moi avec mes coups de griffe. Moi, avec ma passion, mes déchirements, mes conflits avec elle, un contentieux vieux comme le monde. Quand elle m'injurie, je la pervertis, je la désinvestis de son 
arrogance, alors elle me gomme, je l'invente, elle me jette dans l'oubli, ou bien, condescendante, consent à jouer avec moi à mes propres périls. Elle prend peu de risques. Combat sans issue s'il se fait sans compromis, ou sans recours aux barbarismes. Lovée dans ses fonctions quotidiennes reconnues de tous, elle me séduit puis me trahit et m'éconduit. Elle me met en demeure d'écrire dans la demeure seigneuriale ou alors elle me précipite dans le vide. Écris comme ça ou meurs. Je lui tire la langue, je l'étire, je la condense. J'écris, mais autrement. Je reste en vie. (« Langue étrangère» 171)

Bersianik est une amoureuse de la langue qui se résout difficilement à la nécessité de la subvertir, comme on peut le remarquer par les multiples tensions qui traversent cet extrait. Le corps-à-corps avec la langue est présenté comme une liaison amoureuse, une relation difficile qui se joue aux dépens de la femme du couple. On notera au passage l'évocation à regret du compromis et du recours aux barbarismes, seules issues possibles d'un combat à finir.

Le pari de Bersianik au sujet de la langue est d'arriver à s'y inscrire « légalement » en tant que femme. Par « légalement », je paraphrase l'auteure elle-même et souligne du même coup le caractère " peccamineux » de ses stratégies de subversion de la langue. Celles-ci, quoique de portée modérée au sein du texte, y pullulent littéralement. Parmi les plus remarquables figurent sans conteste les multiples déformations orthographiques et autres mots-valises que l'auteure affectionne tout particulièrement : «La fesselle, les enfaons, le lafâge, ce n'est pas notre affaire disent-ils [les hommes]. » (L'Euguélionne 286). Dans cet exemple, les $f$ qui ne sont pas à leur place réussissent habilement à évoquer l'association habituelle de la femme avec la vaisselle, les enfants et le lavage tout en montrant, par l'effet de fausseté qui s'en dégage (ces mots n'existant pas), l'arbitraire d'une telle association. Le tout est d'arriver à développer une relation d'étrangeté du ou des mots visé(s) avec leur(s) signification(s) habituelle(s). L'effet recherché est évidemment une prise de conscience, mais également, (pourquoi pas ?) un sentiment d'irrévérence : «Nous fûmes prises alors d'une envie phormidable non de phallus mais de phourire » (216). « [L]'extravagance du signifiant » donne lieu à « ce petit orgasme orthographique " (Barthes, Le plaisir du texte 103), qui provoque à la fois le rire et un effet de distanciation. C'est là bien sûr, tout l'enjeu du travail de la langue dans L'Euguélionne: acquérir une vue de l'extérieur. Le 
mot est vidé de son sens par un jeu sur le signifiant qui va relativiser le signifié et le placer en position de questionnement par rapport à la lectrice ou au lecteur. Les multiples déformations orthographiques et mots-valises qu'on retrouve un peu partout dans L'Euguélionne représentent un de ces fils « translinéaires » du texte, nous enjoignant à développer un sens critique par rapport à la langue. En détruisant certaines habitudes de lecture, en produisant de nouvelles possibilités d'association des mots entre eux, l'auteure induit un mouvement et des déplacements au sein de la langue qui mettent en valeur l'arbitraire et l'aspect construit non seulement de la grammaire et de la sémantique françaises, mais également des discours fondateurs de l'humanité. C'est le lien entre signifiant et signifié qui est ici remis en question.

Bersianik porte d'ailleurs beaucoup d'attention à ce lien qui unit l'image d'un mot et son sens. L'Euguélionne est truffé de retours à l'étymologie. Ces démonstrations sont faites selon deux objectifs principaux : essais de filiation ou de création. Les premiers visent à montrer les relations d'un mot à d'autres en remontant à ses origines :

Femmes et Hommes de la Terre, dit l'Euguélionne, je suis sûre que les futures générations de cette planète auront assez de santé pour s'amuser à vos dépens quand elles sauront tout sur la carrière du mot fécondation à travers vos civilisations, à commencer par sa descendance. Car vous ne l'ignorez pas, ce mot est à l'origine du mot femme, au même titre que les doux noms de foetus, fellatio et félicité! » (248)

En montrant d'où un mot tire sa substance, l'auteure lève le voile sur les sens cachés qui révèlent sa vraie nature : « Et qu'est-ce que la famille, dit l'Euguélionne? Elle a son origine dans l'esclavage. Le mot "famille" vient du mot "serviteur"! » (301). Pour ce qui est de l'étymologie utilisée dans un objectif de création, l'Euguélionne la requiert lorsque surgit la question de la féminisation des noms professionnels : « vous pouvez chercher dans l'étymologie du français à former vous-mêmes les substantifs féminins propres à vous désigner dans vos professions » (230). Pour elle, le recours à l'étymologie représente un moyen efficace pour créer de nouveaux substantifs féminins en évitant l'écueil d'une ressemblance trop grande (qui deviendra forcément péjorative) avec les noms professionnels masculins. Tout le «tableau des féminins en formation » (231) est basé sur l'étymologie, ce qui atteste l'ampleur du travail de défrichage que s'est imposé l'auteure. 
Il est cependant permis de $s^{\prime}$ interroger sur la pertinence du recours à l'étymologie dans L'Euguélionne en ce qui concerne les essais de création. En effet, si les langues ont de tout temps été créées par et pour les hommes, reculer dans le passé pour former de nouveaux mots dénués de valeur négative à l'endroit des femmes ne semble guère une solution possible. Bersianik aurait-elle commis une « erreur » de raisonnement? C'est peu probable, vu le haut niveau de conscience de l'écrivaine par rapport à sa pratique d'écriture. J'aurais plutôt tendance à rapprocher ce paradoxe des conséquences de l'esthétique du compromis qui sous-tend L'Euguélionne. En demeurant dans la logique de sa propre pensée, Bersianik ne peut travailler sans un ancrage quelconque dans le culturel. Si elle veut que ses créations passent un jour dans l'usage, elle ne peut absolument pas passer outre l'exigence d'un lien conscient avec le passé. Les femmes doivent parvenir à s'inscrire dans une tradition renouvelée par leurs apports multiples sans tomber dans le piège de la ghettoïsation ou de la tradition parallèle. Le français, langue dérivée du latin, n'offre pas le recul nécessaire à l'établissement d'une terminologie dénuée de toute valeur négative à l'endroit des femmes. Afin d'éviter les sens cachés des mots (comme on a vu dans l'exemple de famille), Bersianik remonte à leurs racines. Son entreprise est commandée par un objectif de relexification s'opérant par une mise à nu suivie d'une recharge sémantique des mots en question. Ce mouvement correspond à l'articulation de l'écriture avec la contrainte du culturel.

Et le culturel il est ... c'est le matériel qui fait que je vais pouvoir écrire parce que si je n'avais pas appris à écrire ou si je n'avais pas été enseignée dans différents réseaux culturels, je ne pourrais pas écrire. Mais ce matériel-là même fait frein à ma création parce qu'il me dirige dans des reproductions d'idées ou d'images, alors que moi je veux produire du nouveau. Donc c'est momentané ça. C'est de se vider, se vider soi-même. Mais en me vidant moi-même ... je vide le mot de son sens. Il est vidé de toute façon par la circulation, depuis des siècles le mot a été vidé de son sens, il a évolué : il n'est plus ce qu'il voulait dire. Là on fait vraiment une mise à nu du mot et après ça on le recharge de sens. C'est comme un peu recharger les batteries. («Transcription » 4)

Le recours à l'étymologie constitue donc un autre de ces réseaux textuels " translinéaires » qui contribuent à renforcer la cohérence et la cohésion 
interne du roman. Ceux-ci ont un rôle central à jouer dans la structuration du texte, établissant des liens entre les diverses parties, chapitres, "versets », etc., par de multiples renvois à la fois lisibles et/ou visibles. On trouve également, au nombre de ces réseaux (beaucoup trop nombreux pour que j'aie la prétention de les épuiser ici), la thématique des noms grecs, qui selon Bersianik, est un fil qui « tricote le texte » (Smith 84), ou encore, un ensemble de maladies qui affectent la population mâle de la planète : "l'Engendrite à sens unique " (L'Euguélionne 223), la " nombrilite aiguë » (265), l'« embryonnite aiguë » (270), etc. L'effet cocasse de ces noms farfelus ouvre la porte à l'irrévérence face à l'autorité.

L'irrévérence est bien la notion-clé avec laquelle il faut aborder L'Euguélionne. À travers son roman à succès, celle-ci se lance ouvertement dans une vaste entreprise de démystification et de remise en question des figures de l'autorité patriarcale et des discours qui les représentent. La question du langage est intimement liée à la critique sociale chez Bersianik et devient rapidement l'objet d'un travail minutieux de l'écrivaine pour arriver à y inscrire le féminin. Cette inscription ne peut toutefois se faire n'importe comment. Prise à la fois entre les exigences du genre et ses propres intentions, l'auteure doit se tourner vers des stratégies «peccamineuses» de détournement linguistique afin de produire un travail de subversion de la langue qui soit aisément accessible à toutes et tous. Cette préoccupation, jointe au recours fréquent à la parodie, sont responsables de la préservation des cadres de références du lectorat, dont la déconstruction au sein du texte engendre une prise de conscience chez la lectrice ou le lecteur. Ce mode de fonctionnement est le foyer du didactisme de L'Euguélionne. Le discours métalinguistique et ses répercussions sur la forme sont pensés en fonction d'un objectif d'éducation de la population, sexes confondus (même si on ne s'illusionne pas sur la prédominance des femmes dans le lectorat de Bersianik). Sinon, comment expliquer la présence de tableaux (226-27, 231-34, 250), de démonstrations stylistiques $(227,250)$, de listes et d'énumérations (139-44, 152-53, 155-59) ? Le travail minutieux à partir du dictionnaire s'inscrit en droite ligne avec l'esprit général du roman : se servir du culturel et de l'appris afin de mieux les subvertir.

Au niveau des répercussions du discours métalinguistique sur la forme dans L'Euguélionne, le déséquilibre initial qu'on perçoit à la lecture de ces phrases élégantes, jamais alambiquées et respectant le plus souvent les règles et l'esprit de la langue, est compensé dans une certaine mesure par le foisonnement des réseaux textuels et du travail sur le signifiant. 
L'auteure multiplie les efforts afin de faire résonner les mots dans toute l'ampleur de leur histoire ( $\mathrm{d}$ 'où le recours fréquent à l'étymologie) et créer une polyphonie et une polysémie qui provoqueront une prise de conscience chez la lectrice ou le lecteur. C'est d'ailleurs autour de la relation étroite qui se développe entre l'auteure et son lectorat que L'Euguélionne est construit. C'est également à la lumière de cette relation particulière qu'il faut comprendre, à mon avis, l'esthétique du compromis qui règne dans le roman, où le jeu textuel cède parfois le pas au didactisme le plus évident. La portée du discours de L'Euguélionne s'arrête au seuil de la conscience. Mon propos ici n'est pas de condamner L'Euguélionne pour sa « lisibilité », caractéristique peu fréquente des productions littéraires des féministes des années soixante-dix. En accentuant, au cours de cet article, le débalancement que j'ai repéré entre la forme et le contenu de L'Euguélionne, j’ai voulu montrer à quel point son rapport avec la norme masculine de domination du langage, quoique clairement situé du côté de la transgression au niveau discursif, devient problématique lorsque la langue et l'écriture littéraire imposent des compromis à l'écrivaine qui doit parfois céder à contrecœur à leur tyrannie. Prise à la fois dans le langage et dans les réseaux culturels qui font de nous ce que nous sommes, l'écrivaine qui désire inscrire sa subjectivité de femme dans la langue ne peut le faire qu'à partir de sa position inconfortable dans les marges de l'ordre symbolique, produisant ainsi une écriture fascinante de par les multiples tensions qui la traversent.

\section{Notes}

Cet article est une version condensée et adaptée du premier chapitre de mon mémoire de maîtrise, intitulé Discours métalinguistique et pratiques d'écriture féministes (2000) et réalisé à l'aide d'une bourse de recherche du Fonds FCAR.

${ }^{1}$ Louky Bersianik, La main tranchante du symbole (Montréal : Les Éditions du Remue-ménage, 1990) 47. Désormais, les renvois à ce livre seront indiqués par le sigle $M T$.

${ }^{2}$ Ceci n'est évidemment pas sans rappeler les théories d'Edwin Ardener à propos des «mutant/dominant groups ». Voir à ce sujet l'introduction de Shirley Ardener dans Perceiving Women, xiii-xiv, plus particulièrement. ${ }^{3}$ Alain Rey (dir.), Le Robert. Dictionnaire historique de la langue française (Paris : Dictionnaire le Robert, 1998) 2627. 
${ }^{4}$ Mary Daly, "Theology after the demise of God the father : a call for the castration of sexist religion " (130), cité dans Dale Spencer, Man Made Language, 167.

${ }^{5}$ Voir Karen Gould, Writing in the Feminine : Feminism and Experimental Writing in Quebec, 160.

\section{Ouvrages cités}

Ardener, Shirley. «Introduction ». Perceiving Women. London: Malaby, 1975.

Barthes, Roland. Le degré zéro de l'écriture. Paris : Seuil, 1972.

—. Le plaisir du texte. Paris : Seuil, 1973.

Bersianik, Louky. « Entrevue : Louky Bersianik. Entre la dictée de l'inconscient et le tremblement de la conscience ». Arcade 2 (1986).

—. L'Euguélionne. Montréal : La Presse, 1976.

—. "Langue étrangère en quête d'auteure ». Lise Gauvin. « Du bruit au bruissement : à propos de langue et d'écriture ». Possibles XI.3 (1987).

- La main tranchante du symbole. Montréal : Les Éditions du Remueménage, 1990.

—. « La mémoire courte ». La vie en rose (juillet/août 1985).

—. « Noli mi tangere ». La Barre du jour 56-57 (mai/août 1977).

—. «Transcription d'une emission de radio ». 17 février 1982. Montréal : Les entreprises Radio-Canada.

Bourdieu, Pierre. La domination masculine. Paris : Seuil, 1998.

Forsyth, Louise. «L'écriture au féminin : L'Eugélionne de Louky Bersianik, L'Absent aigu de Geneviève Amyot, L'Amer de Nicole Brossard ». Journal of Canadian Fiction 25-26 (1979).

Gould, Karen. Writing in the Feminine : Feminism and Experimental Writing in Quebec. Carbondale : Southern Illinois UP, 1990.

Lacan, Jacques. Encore. Le séminaire - Livre XX. Paris : Seuil, 1999.

Smith, D. "Louky Bersianik et la mythologie du futur : de la théorie-fiction à l'émergence de la femme positive ». Lettres québécoises 27 (automne 1982).

Spencer, Dale. Man Made Language. London: Routledge and Kegan Paul, 1985. 